\title{
Nanoscale
}

PAPER
Check for updates

Cite this: Nanoscale, 2017, 9, 9175

Received 9th May 2017,

Accepted 15th June 2017

DOI: $10.1039 / c 7 n r 03297 h$

rsc.li/nanoscale

\section{Long-pulse laser launch and ionization of tailored large neutral silver nanoparticles with atomic mass assignment $\dagger$}

\author{
A. Gallego, ${ }^{a}$ U. Sezer, ${ }^{b}$ M. Arndt (DD ${ }^{b}$ and M. Mayor (D) *a,c,d
}

We explore the synthesis, characterization, neutral launch and vacuum ultraviolet ionization of massive perfluorinated-alkyl-capped nanoparticles. The presence of the ligand coating in solution is corroborated by Fourier transform infrared spectroscopy (FT-IR) and the particle size distribution is analyzed by transmission electron microscopy (TEM). Matrix-assisted laser desorption/ionization time-of-flight (MALDI-TOF) mass spectrometry identifies perfluoralkyl coated silver nanoparticles as the most stable species among the materials studied here. They can be launched in high vacuum using long-pulse lowpower laser heating - orders of magnitude below typical thresholds for laser desorption. Energy-dispersive X-ray spectroscopy (EDX) of the recaptured silver clusters confirms the expected elemental distribution. Volatilization with subsequent ionization of the neutral nanoparticle beam in high vacuum by $157 \mathrm{~nm}$ light allows analyzing their mass with atomic resolution.

\section{Introduction}

Nanoparticles have moved into the spotlight of research, because their physical and chemical properties are size-dependent and can be tailored by controlling their structure. ${ }^{1,2}$ Metal nanoclusters have a wide spectrum of potential applications in catalysis, ${ }^{3-5}$ nanoelectronics, ${ }^{6,7}$ sensing, ${ }^{8-11}$ nanoprinting ${ }^{12-16}$ and drug delivery. ${ }^{17}$ Functionalization with tailored ligands can provide them with electrical, optical, catalytic, thermal or solubility properties that would be otherwise inconceivable. ${ }^{18-22}$

Several techniques have been developed for volatilizing and characterizing isolated high-mass particles in high vacuum: electrospray ionization (ESI) has been used to generate highly charged beams of ligand-stabilized nanoclusters in the gas phase. ${ }^{23-25}$ However, applications in soft surface deposition, ${ }^{26}$ laser induced forward transfer, ${ }^{27}$ classical deflectometry or fundamental tests of quantum physics ${ }^{28}$ can profit from beams of neutral and slow nanoparticles. Common volatiliz-

\footnotetext{
${ }^{a}$ University of Basel, Department of Chemistry, Basel, 4056, Switzerland. E-mail: marcel.mayor@unibas.ch

${ }^{b}$ University of Vienna, Faculty of Physics, Vienna, 1090, Austria

${ }^{c}$ Karlsruhe Institute of Technology, Institute for Nanotechnology, 76021 Karlsruhe, Germany

${ }^{d}$ Lehn Institute of Functional Materials (LIFM), Sun Yat-Sen University (SYSU), XinGangXi Rd. 135, 510275 Guangzhou, P.R. China

$\dagger$ Electronic supplementary information (ESI) available: Synthesis and characterization of the nanoclusters: FT-IR, TGA, MALDI-ToF and signal $v s$. desorption study (PDF file). See DOI: 10.1039/c7nr03297h
}

ation methods (MALDI, ESI) require elaborate techniques to slow down the charged nanoparticles to ensure low kinetic energies for soft landing as higher energies will lead to substantial deformation of nanoparticles during the impact. Low velocities are also a prerequisite for successful fundamental quantum experiments, as the particle's momentum is inversely proportional to its de Broglie wavelength. Techniques like laser desorption ionization (LDI) without the use of an additional matrix have shown already that the molecular beam velocity can be drastically reduced. ${ }^{29,30}$

Evidence has recently been provided for the thermal transfer of neutral silicon nanoparticles between two surfaces across a short distance in ultrahigh vacuum. ${ }^{31}$ Subsequent atomic force microscopy allowed imaging the re-captured silicon with core diameters around $2 \mathrm{~nm}$ covered by a thin soft shell. Here, we show that mass spectrometry of thermally launched neutral particles can furthermore provide the unambiguous identification of the attached ligands in the gas phase, with atomic resolution. We achieve this using soft photo-ionization in the gas phase even for masses up to several $10 \mathrm{kDa}$. We test this idea for various combinations of cluster cores and ligand shells and find, that the successful launch, ionization and detection strongly depends on the specific nature of the particle core and ligand composition. Based on successful experiments with carbon nanospheres ${ }^{32,33}$ and massive libraries of porphyrin derivatives, ${ }^{30,34}$ we have explored the possibility of synthesizing a library of silver nanoparticles capped by perfluoroalkyl chains. The electronegative fluorine atoms withdraw electron density from the alkyl 
chains, reduce their polarizability and lower the attractive van der Waals interaction between neighboring nanoparticles. This should facilitate the volatilization even of compounds of considerable size. The metal cluster core shall add mass, define the optical response and enable soft photoionization by vacuum ultraviolet (VUV) radiation for subsequent mass analysis of the free-flying neutral nanoparticles. Inspired by this vision, we here report on the synthesis, launch and ionization of such tailored nanoclusters.

\section{Experimental results}

\section{Nanoparticle synthesis}

Even though a large range of metals is amenable to functionalization, it is not a priori obvious which material-ligand combination may offer the highest stability ${ }^{35}$ during thermal launch and the highest detectability in laser-induced ionization after volatilization. In a preliminary search, we synthesized nanoparticles of gold, platinum, silicon, silver and silver-sulfur which we coated with different aromatic and/or fluorinated ligands in order to assist the ionization and volatilization processes, respectively (see Fig. S1 $\dagger$ ). The particles were characterized and analyzed by MALDI-ToF mass spectrometry. In most of the cases, low-intensity broad bands were detected due to fragmentations or aggregations of the particles under MALDI conditions. A well-resolved spectrum was obtained with platinum particles coated with 2-phenylethanethiol, however its detailed analysis revealed $\mathrm{C}-\mathrm{O}$ bond cleavage during MALDI which left ligand-free platinum oxide clusters behind (ESI Fig. S1†). The desired behavior was finally found for silver nanoparticles coated with alkylthiols exposing perfluorinated tails. While a Brust-Schiffrin ${ }^{36,37}$ approach could not attach the ligands to the metal cores, the polyol-method ${ }^{38,39}$ was successful. Details in the synthesis are crucial for the reproducibility of the procedure and strongly influence the physico-chemical behavior of the particles. However, once prepared, samples maintained reproducible properties over at least two years.

To explore the reactivity of the terminal thiol group we selected alkylthiols of various lengths.

We synthesized particles capped by 3,3,4,4,5,5,6,6,6-nonafluoro-1-hexanethiol ( $\left.\mathrm{L}^{(1)}=\mathrm{SC}_{2} \mathrm{H}_{4} \mathrm{C}_{4} \mathrm{~F}_{9}\right), 3,3,4,4,5,5,6,6,7,7,8,8,8$ tridecafluoro-1-octanethiol $\left(\mathrm{L}^{(2)}=\mathrm{SC}_{2} \mathrm{H}_{4} \mathrm{C}_{6} \mathrm{~F}_{13}\right)$, and $3,3,4,4,5,5,6,6,7,7,8,8,9,9,10,10,10$-heptadecafluoro-1-decanethiol $\left(\mathrm{L}^{(3)}=\mathrm{SC}_{2} \mathrm{H}_{4} \mathrm{C}_{8} \mathrm{~F}_{17}\right)$ to yield the particles $\mathbf{F}_{\mathbf{9}}$-AgNP1, $\mathbf{F}_{\mathbf{1 3}}$-AgNP2 and $\mathbf{F}_{\mathbf{1 7}}$-AgNP3, respectively. The non-fluorous analogue $\mathbf{H}_{\mathbf{1 7}}$ AgNP4 was produced using octanethiol $\left(\mathrm{L}^{(4)}=\mathrm{SC}_{2} \mathrm{H}_{4} \mathrm{C}_{6} \mathrm{H}_{13}\right)$ and served as a reference sample. All four nanoparticles were synthesized by heating silver nitrate and two equivalents of the thiol ligand in ethylene glycol at $160{ }^{\circ} \mathrm{C}$ for 20 hours (Scheme 1). They were purified through cycles of centrifugationredispersion in methanol, THF and diethyl ether.

\section{Verification of ligand attachment in solution}

Infrared spectroscopy on purified silver nanoparticles retrieved all absorption bands of the free ligands, only slightly shifted

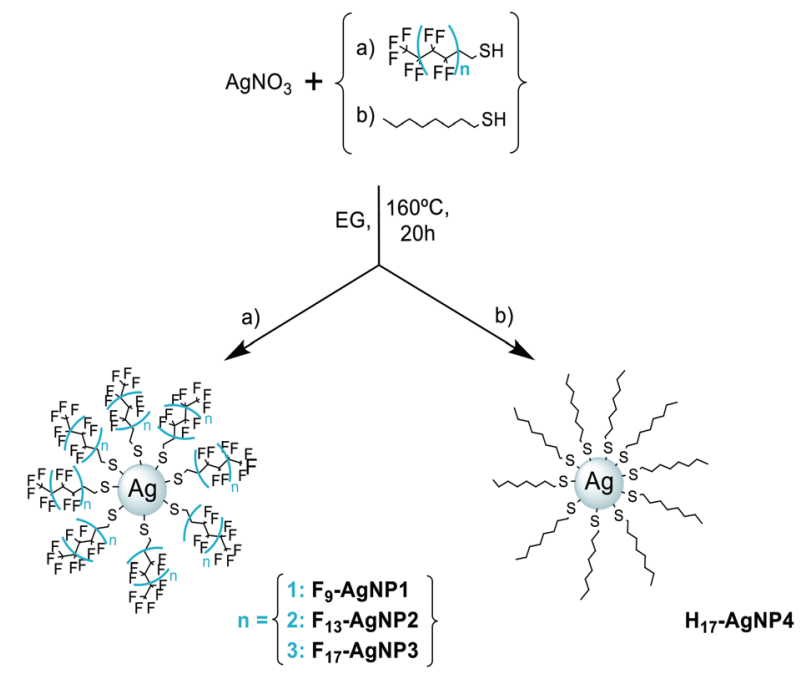

Scheme 1 Synthesis of silver nanoparticles decorated by three different perfluorinated ligands: $\mathrm{F}_{9}$-AgNP1 ( $\left.\mathrm{L}^{(1)}=\mathrm{SC}_{2} \mathrm{H}_{4} \mathrm{C}_{4} \mathrm{~F}_{9}\right), \mathrm{F}_{13}$-AgNP2 $\left(\mathrm{L}^{(2)}=\mathrm{SC}_{2} \mathrm{H}_{4} \mathrm{C}_{6} \mathrm{~F}_{13}\right)$ and $\mathrm{F}_{17}-\mathrm{AgNP3}\left(\mathrm{L}^{(3)}=\mathrm{SC}_{2} \mathrm{H}_{4} \mathrm{C}_{8} \mathrm{~F}_{17}\right)$, to study the role of the ligand length, and with the non-fluorous octanethiol $\left(\mathrm{L}^{(4)}=\right.$ $\mathrm{SC}_{2} \mathrm{H}_{4} \mathrm{C}_{6} \mathrm{H}_{13}$ ) on $\mathrm{H}_{17}-\mathrm{AgNP} 4$ to verify the importance of fluorination.

towards higher energy. Fig. 1 compares both spectra for $\mathbf{F}_{\mathbf{1 7}}$ AgNP3. This corroborates that they were stably attached to the metal cores. Similar spectra have been recorded for all nanoparticles types in this study (see ESI Fig. S2 $\dagger$ ).

A subsequent thermogravimetric analysis (TGA) of the same batch allowed us to determine the molar ratio of silver atoms and ligands (see ESI Fig. S3 $\dagger$ ) to be 2.3 for $\mathbf{F}_{\mathbf{9}}$-AgNP1, 1.5 for $\mathbf{F}_{13}$-AgNP2, 2.3 for $\mathbf{F}_{17}$-AgNP3, and 2.0 for $\mathbf{H}_{17}$-AgNP4.

TEM images (Fig. 2) showed well isolated nanoparticles with core diameters between $0.5-1 \mathrm{~nm}$ varying by up to $40 \%$ within one sample. Such polydispersity is common in colloidal nanoparticles. ${ }^{40}$

To understand their underlying atomic composition, we subjected all species to MALDI-ToF mass spectrometry recording all spectra in the positive mode (ESI Fig. S4†). A zoom into

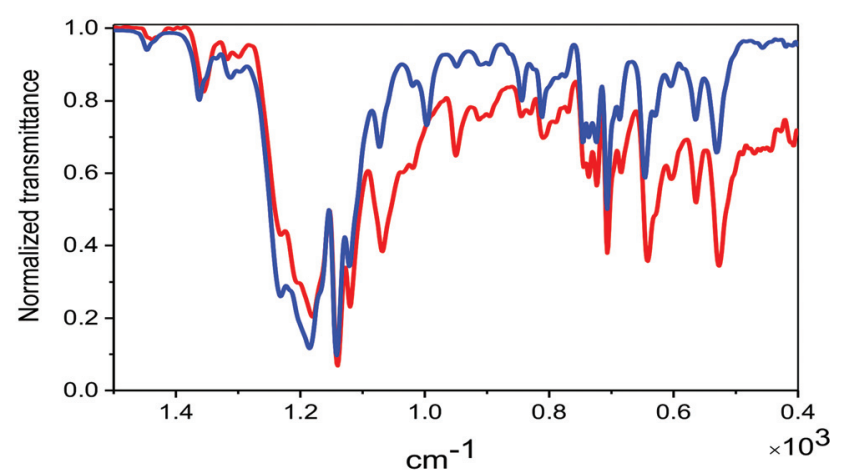

Fig. 1 Infrared spectrum of the free ligands (blue line) and the silver nanoparticle $\mathrm{F}_{17}$-AgNP3 (red line). The purified nanoparticles display all vibrational bands of the isolated ligands, indicating their intact attachment to the silver core. 

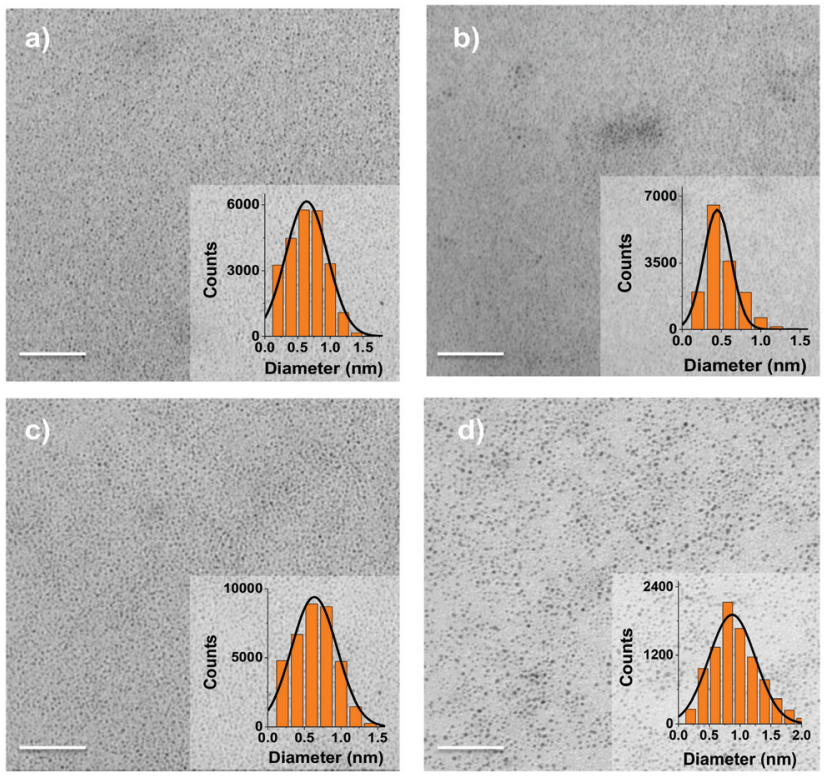

Fig. 2 Transmission electron micrographs of ligand-stabilized silver nanoparticles. Particles in solution are dropped onto a graphene TEM grid, dried and imaged. The mean particle diameters and their standard deviation are (a) $F_{9}$-AgNP1 $d=(0.7 \pm 0.3) \mathrm{nm}$, (b) $F_{13}-A g N P 2 d=(0.5 \pm$ $0.2) \mathrm{nm}$, (c) $\mathrm{F}_{17}-\mathrm{AgNP3} d=(0.7 \pm 0.3) \mathrm{nm}$ and (d) $\mathrm{H}_{17}-\mathrm{AgNP} 4 d=(1 \pm$ 0.4) $\mathrm{nm}$. The scale bar corresponds to $50 \mathrm{~nm}$.

the low mass range up to $10000 \mathrm{Da}$ (Fig. 3) shows a series of equidistant peaks consistently separated by the mass of exactly one ligand and one silver atom, i.e. by $388 \mathrm{Da}$ for $\mathrm{AgL}^{(1)}$ in $\mathbf{F}_{\mathbf{9}^{-}}$ AgNP1, 488 Da for $\mathrm{AgL}^{(2)}$ in $\mathbf{F}_{\mathbf{1 3}}$-AgNP2, $588 \mathrm{Da}$ for $\mathrm{AgL}^{(3)}$ in $\mathbf{F}_{\mathbf{1 7}}$-AgNP3 and 256 Da for $\mathrm{AgL}^{(4)}$ in $\mathbf{H}_{\mathbf{1 7}}$-AgNP4.

\section{Thermal launch and VUV ionization}

The silver nanoparticles were then exposed to thermal laser heating and the emerging plume subjected to VUV ionization in high vacuum (Fig. 4). A blue diode laser, gated to a pulse
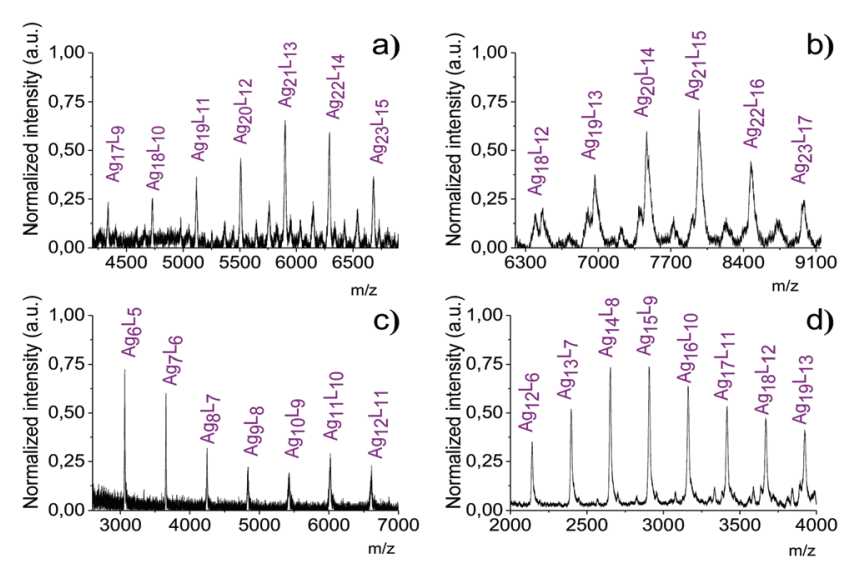

Fig. 3 MALDI mass spectra of the Nanoparticles (a) $F_{9}$-AgNP1, (b) $F_{13}$ AgNP2, (c) $\mathrm{F}_{17}-\mathrm{AgNP3}$ and (d) the non-fluorous nanoparticles $\mathrm{H}_{17^{-}}$ AgNP4 and the assigned molecular formulas of the species within the cluster library.

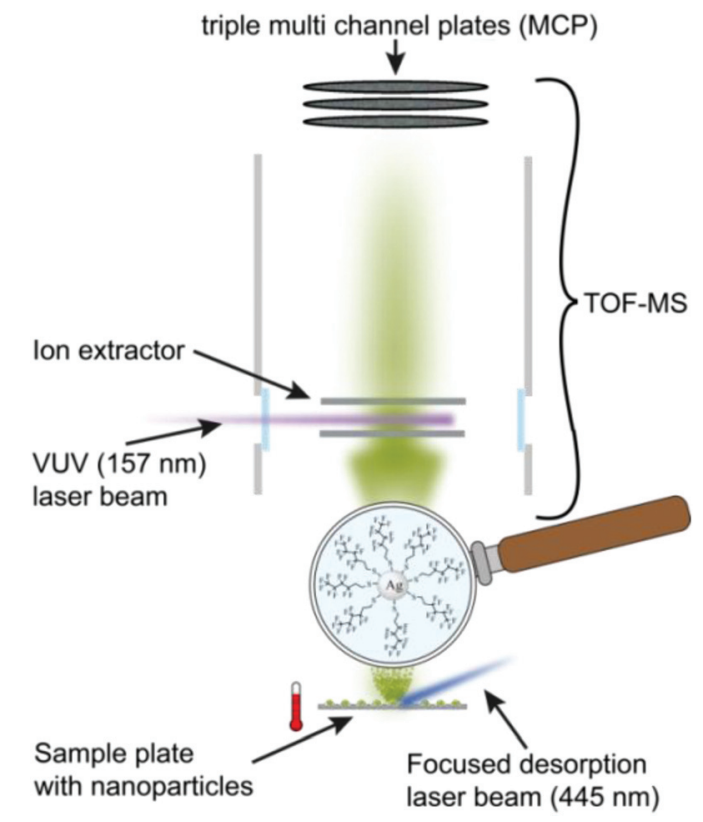

Fig. 4 Launch, recapture and VUV ionization of perfluoroalkyl-capped silver nanoparticles. The particles were launched by a long-pulse (10 ms) laser beam (445 nm, electrically chopped, max. $3 \mathrm{~W} \mathrm{cw}, 2 \mathrm{~mm}$ spot diameter). In recapture experiments the particles were collected on a carbon grid $4 \mathrm{~cm}$ above the launch pad and later analyzed ex situ in scanning electron microscopy. For mass analysis, particles were ionized by a vacuum ultraviolet (VUV) laser beam (157 nm, $E \leq 1.1 \mathrm{~mJ}, A=1 \times$ $3 \mathrm{~mm}$, pulse length $10 \mathrm{~ns}$ ) and detected in time-of-flight mass spectrometry $(m / \Delta m \approx 100)$.

length of $10 \mathrm{~ms}$ and a pulse energy of up to $E=30 \mathrm{~mJ}$, was focused onto the nanoparticles that had been prepared as a thin layer on a metal plate. The laser intensity of up to 95 $\mathrm{W} \mathrm{cm} \mathrm{cm}^{-2}$ was 4-5 orders of magnitude below threshold for MALDI-like laser desorption $\left(10^{6}-10^{7} \mathrm{~W} \mathrm{~cm}^{-2}\right)$.

In a first set of experiments, we heated the nanoparticles in the blue laser beam and captured them on a carbon grid $4 \mathrm{~cm}$ above. EDX in scanning electron microscopy allowed us to identify the atomic content of the collected material. Starting from $\mathbf{F}_{\mathbf{1 3}}$-AgNP2 particles, we found silver, carbon and fluorine. In contrast to that, the laser-induced transfer of $\mathbf{H}_{\mathbf{1 7}}-\mathbf{A g N P 4}$ resulted in a pure carbon film, sparsely decorated with particles up to several hundred nanometers in diameter, containing silver and sulfur but hardly any carbon. This indicates that the cores separated from their ligands and aggregated to metal-sulfide clumps.

To characterize the atomic composition of the nanoparticles in free flight, we have intersected them with an $F_{2}$ laser beam. The resulting ions were analyzed in a linear timeof-flight mass spectrometer. All nanoparticles were tested under the same volatilization conditions and we verified the initial neutrality of the evaporated beam: ions could only be detected in the presence of ionizing VUV light. Its intensity was optimized for each species to maximize their signal and minimize their photo-fragmentation. 
Clean and atomically resolved mass spectra could only be acquired for $\mathbf{F}_{\mathbf{9}}$-AgNP1 and $\mathbf{F}_{\mathbf{1 3}}$-AgNP2. Fig. 5a shows three different libraries within the mass distribution of $\mathbf{F}_{\mathbf{9}}$-AgNP1, marked by dots, stars and triangles. For $\mathbf{F}_{\mathbf{1 3}}$-AgNP2, we identify at least five different cluster libraries (Fig. 5b).

They differ in their basic $\mathrm{Ag}_{x} \mathrm{~L}_{y}$ core but within the same library, the nanoparticles differ consistently between next neighbors in the mass spectrum by one $\mathrm{AgL}^{(1)}$ for $\mathbf{F}_{\mathbf{9}}$-AgNP1 and one $\mathrm{AgL}^{(2)}$ for $\mathbf{F}_{\mathbf{1 3}}$-AgNP2. This is consistent with our previous MALDI-ToF spectra and with the hypothesis that the nanoparticles are launched intactly, surrounded by their ligand chains.

$\mathbf{F}_{13}$-AgNP2 had higher signal than $\mathbf{F}_{\mathbf{9}}$-AgNP1 even though the particles are more massive. This confirms the importance of a well-tailored perfluoroalkyl coating. Consistent with this hypothesis, we could not detect any sizeable signal for the non-fluorous $\mathbf{H}_{\mathbf{1 7}}$-AgNP4 nanoparticles. Surprisingly, we were not able to record any ionization mass spectrum of $\mathbf{F}_{\mathbf{1 7}}$-AgNP3, the particle with the longest perfluorinated chains of all four test objects.

Since $\mathbf{F}_{\mathbf{1 7}}$-AgNP3 particles seem to be susceptible to laser induced transfer in high vacuum, as verified by TEM imaging, we hypothesize that electron recapture may suppress the ionization probability. It therefore seems that the $3,3,4,4,5,5,6,6,7,7,8,8,8$-tridecafluoro-1-octanethiol ligand of F $_{13}$-AgNP2 represents a good compromise in chain length between facilitating volatilization and not preventing ionization.

We note that high ionization laser intensities may also cause fragmentation and thus down-shift the mass spectra (see ESI Fig. S5 $\dagger$ ). When we gradually increase the ionization intensity by a factor of five from $8.5 \times 10^{5}$ to $4 \times 10^{6} \mathrm{~W} \mathrm{~cm}^{-2}$ the detected ion distribution shifts to lower masses and the peaks are less well resolved. However, at optimized detection laser intensity the multimodal character of the mass spectra is

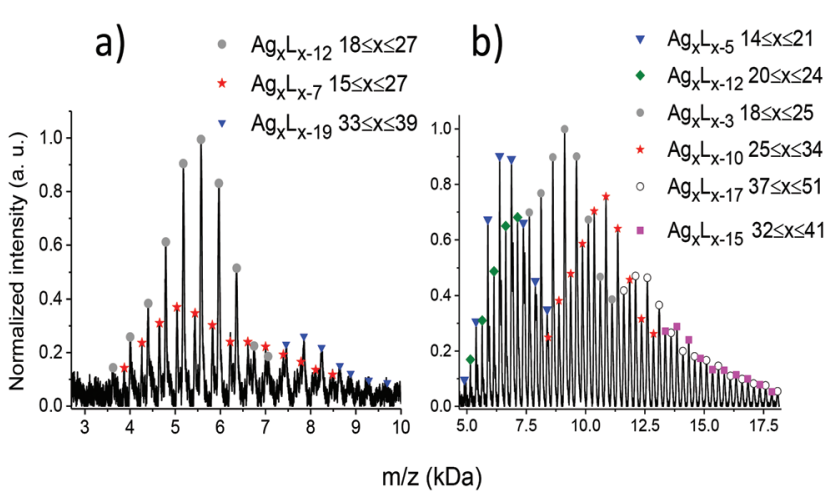

Fig. 5 Vacuum ultraviolet photoionization mass spectra after ms-laser heating. The peak separation within every series corresponds to AgL, the mass of one ligand and one silver atom. (a) $\mathrm{F}_{\mathbf{9}}$-AgNP1 library; VUV laser intensity is $(85 \pm 4) \times 10^{4} \mathrm{~W} \mathrm{~cm}^{-2}$, signal electronically amplified by $\times 50$ (b) $\mathrm{F}_{13}$-AgNP2 signal, without electronic amplification; the ionization intensity was $(58 \pm 4) \times 10^{4} \mathrm{~W} \mathrm{~cm}^{-2}$. The different nanoparticle libraries were identified and are labelled with blue triangles, stars, rhombuses, circles (empty \& filled) and squares. present both in MALDI and in the post-volatilization data. This corroborates the assumption that the long-pulse neutral launch largely maintains the integrity of the original particles.

\section{Discussion}

It may seem surprising that even nanoparticles in the mass range up to $20000 \mathrm{Da}$ can be volatilized using millisecond laser heating, at light intensities far too low for typical laser desorption. Preliminary experiments with an extended library of $\mathbf{F}_{\mathbf{1 7}}$-AgNP2, in the mass range between 60000 and 100000 Da show that volatilization by slow laser heating can even be extended to such highly massive particles - although at reduced signal strength and without atomic mass resolution.

A tentative explanation for the underlying mechanism may be found in analogy to a recent study where perfluoroalkylated gold nanoparticles were used as a decomposing matrix to softly launch small biomolecules: a nanosecond UV laser pulse detached the ligands from the gold particles and provided an expanding perfluorinated matrix to launch the analyte biomolecules. ${ }^{41}$

Here we propose that a similar mechanism may also be at work, however with the analyte particles providing their own matrix and the nanosecond laser pulse being replaced by millisecond laser heating. Earlier studies using microfocus laser sources showed that at laser intensities of $3 \mathrm{MW} \mathrm{cm} \mathrm{cm}^{-2}$ thin layers of dye molecules could be heated to about $1000 \mathrm{~K}$ and evaporated intactly. ${ }^{42}$ In our case, purely thermal evaporation of $10 \mathrm{kDa}$ particles at $200 \mathrm{~m} \mathrm{~s}^{-1}$ could only be explained by assuming a temperature of $36000 \mathrm{~K}$. This temperature is very unlikely, since we know from thermogravimetric analysis that perfluoroalkyl-capped silver nanoparticles in the mass range of $10 \mathrm{kDa}$ will decompose when exposed to $520 \mathrm{~K}$ for a long period of time. In comparison to that, a self-seeded matrix process seems plausible: long-pulse laser desorption is likely to release a locally dense gas of perfluoroalkyl chains from a subset of clusters and these chains propel another subset of silver nanoparticles intactly into the gas phase, which may even be surprisingly massive. Their beam velocity is then determined by the mass and energy of the ligand plume that acts as a slow carrier gas, leading to a more realistic launch temperature around $1000 \mathrm{~K}$. This mechanism resembles MALDI in that small molecules can launch surprisingly large nanoparticles largely intactly. However, our studies were only successful using long-pulse low-intensity radiation and consistently failed when using nanosecond pulses of the same energy of several $\mathrm{mJ}$.

This observation raises the question whether the ligand coated nanoparticles observed in postionization mass spectrometry may have aggregated in the desorption plume and not represent the synthesized sample. To elucidate this question, a mixture of $\mathbf{F}_{9}$-AgNP1 and $\mathbf{F}_{13}$-AgNP2 was launched, ionized and detected following the same procedure as before. If the fragmentation-aggregation model were correct, both ligands $\mathrm{L}^{(1)}$ and $\mathrm{L}^{(2)}$ should finally be found on the same par- 
ticle. However, we find only peak distances of around $490 \mathrm{Da}$ (ESI Fig. S6 $\dagger$ ) corresponding to the ligands of $\mathbf{F}_{\mathbf{1 3}} \mathbf{- A g N P 2} \cdot \mathbf{F}_{\mathbf{9}^{-}}$ AgNP1 ligands never appear on $\mathbf{F}_{\mathbf{1 3}}$-AgNP2. $\mathbf{F}_{\mathbf{9}}$-AgNP1 does not appear in the mixed sample either. They are visibly desorbed from the sample plate but are not recorded in the same mass spectrum, since the optimal detection laser pulse energy maximizing the ion signal and minimizing ionization-induced fragmentation - differs by a factor of two between the two nanoparticle species.

\section{Conclusion}

We have presented the synthesis and characterization of a new class of perfluoroalkyl-functionalized silver nanoparticles, and the assignment of their atomic composition using MALDI. We have found that surprisingly massive particles can be launched in high vacuum by millisecond laser heating at low optical intensity. We have proved the presence of massive ligandcapped particles using VUV photoionization mass spectrometry, with unambiguous atomic assignment. The ligand length and chemical composition can be tailored to shape the thermal and ionization properties of the nanoparticles. Soft launch and postionization require a delicate balance between ligand length, composition and core mass. The data are consistent with the assumption that ligand-capped nanoparticles can be launched by the expansion of a self-seeding carrier gas of ligand side chains.

\section{Acknowledgements}

We acknowledge financial support through EU project No. 304886, ERC No. 320694, FWF W1210-N25, the Swiss National Science Foundation (Grant No. 200020_159730) as well as the "Forschungskommission" of the University of Basel. We acknowledge fruitful discussions with Nadine Dörre, Jonas Rodewald, Philipp Geyer, and Armin Shayeghi. We also acknowledge Annika Büttner, Markus Dürrenberger and Stephan Puchegger for their contributions on the TGA, TEM and SEM/EDX studies of the nanoparticles, respectively.

\section{Notes and references}

1 Y. Lu and W. Chen, Sub-nanometre sized metal clusters: from synthetic challenges to the unique property discoveries, Chem. Soc. Rev., 2012, 41, 3594-3623.

2 L. D. Pachón and G. Rothenberg, Transition-metal nanoparticles: synthesis, stability and the leaching issue, Appl. Organomet. Chem., 2008, 22, 288-299.

3 Y. Zhu, H. Qian and R. Jin, Catalysis opportunities of atomically precise gold nanoclusters, J. Mater. Chem., 2011, 21, 6793-6799.

4 G. Li and R. Jin, Atomically precise gold nanoclusters as new model catalysts, Acc. Chem. Res., 2013, 46, 1749-1758.
5 D. W. Goodman, Chemistry: Precious little catalyst, Nature, 2008, 454, 948-949.

6 G. Schmid, Nanoclusters-Building Blocks for Future Nanoelectronic Devices?, Adv. Eng. Mater., 2001, 3, 737743.

7 G. Khomutov, R. Gainutdinov, S. Gubin, V. Kislov, V. Khanin, A. Rakhnyanskaya, A. Sergeev-Cherenkov, E. Soldatov, D. Suyatin and I. Taranov, Organized planar nanostructures from ligand-stabilized nanoclusters: a route to molecular nanoelectronic devices, Appl. Surf. Sci., 2004, 226, 149-154.

8 N. Gupta, D. Gupta, S. Aggarwal, S. Siddhanta, C. Narayana and H. C. Barshilia, Thermally stable plasmonic nanocermets grown on microengineered surfaces as versatile surface enhanced Raman spectroscopy sensors for multianalyte detection, ACS Appl. Mater. Interfaces, 2014, 6, 22733-22742.

9 M. D. Malinsky, K. L. Kelly, G. C. Schatz and R. P. Van Duyne, Chain length dependence and sensing capabilities of the localized surface plasmon resonance of silver nanoparticles chemically modified with alkanethiol selfassembled monolayers, J. Am. Chem. Soc., 2001, 123, 14711482.

10 G. K. Joshi, S. Deitz-McElyea, M. Johnson, S. Mali, M. Korc and R. Sardar, Highly Specific Plasmonic Biosensors for Ultrasensitive MicroRNA Detection in Plasma from Pancreatic Cancer Patients, Nano Lett., 2014, 14, 69556963.

11 N. Kahn, O. Lavie, M. Paz, Y. Segev and H. Haick, Dynamic Nanoparticle-Based Flexible Sensors: Diagnosis of Ovarian Carcinoma from Exhaled Breath, Nano Lett., 2015, 15, 7023-7028.

12 M. Makrygianni, I. Kalpyris, C. Boutopoulos and I. Zergioti, Laser induced forward transfer of $\mathrm{Ag}$ nanoparticles ink deposition and characterization, Appl. Surf. Sci., 2014, 297, 40-44.

13 S. H. Ko, H. Pan, C. P. Grigoropoulos, C. K. Luscombe, J. M. Fréchet and D. Poulikakos, All-inkjet-printed flexible electronics fabrication on a polymer substrate by low-temperature high-resolution selective laser sintering of metal nanoparticles, Nanotechnology, 2007, 18, 345202.

14 G. Melinte, S. Moldovan, C. Hirlimann, X. Liu, S. BéginColin, D. Bégin, F. Banhart, C. Pham-Huu and O. Ersen, Towards nanoprinting with metals on graphene, Nat. Commun., 2015, 6, 8071.

15 U. Zywietz, A. B. Evlyukhin, C. Reinhardt and B. N. Chichkov, Laser printing of silicon nanoparticles with resonant optical electric and magnetic responses, Nat. Commun., 2014, 5, 3402.

16 W. K. Lee, Z. Dai, W. P. King and P. E. Sheehan, Maskless nanoscale writing of nanoparticle- polymer composites and nanoparticle assemblies using thermal nanoprobes, Nano Lett., 2009, 10, 129-133.

17 E. Blanco, H. Shen and M. Ferrari, Principles of nanoparticle design for overcoming biological barriers to drug delivery, Nat. Biotechnol., 2015, 33, 941-951. 
18 J. Wei, N. Schaeffer and M.-P. Pileni, Ag nanocrystals: 1. effect of ligands on plasmonic properties, J. Phys. Chem. $B, 2014,118,14070-14075$.

19 J. Zhou, G. Song, Y. Li, Y. Song, B. Chen, X. Zhang, T. Wang, Y. Fu and F. Li, Thermal Annealing: A Facile Way of Conferring Responsivity to Inert Alkyl-Chain-Passivated Nanoparticle Arrays, Langmuir, 2014, 30, 13052-13057.

20 C. Chen, Z. Hu, Y. Li, L. Liu, H. Mori and Z. Wang, In-Situ High-Resolution Transmission Electron Microscopy Investigation of Overheating of Cu Nanoparticles, Sci. Rep., 2016, 6, 19545.

21 S. Raza, S. Kadkhodazadeh, T. Christensen, M. Di Vece, M. Wubs, N. A. Mortensen and N. Stenger, Multipole plasmons and their disappearance in few-nanometre silver nanoparticles, Nat. Commun., 2015, 6, 8788.

22 K. Kroy, Levitating nanoparticles: Non-equilibrium nanothermometry, Nat. Nanotechnol., 2014, 9, 415-417.

23 A. R. M. Bustos, J. R. Encinar and A. Sanz-Medel, Mass spectrometry for the characterisation of nanoparticles, Anal. Bioanal. Chem., 2013, 405, 5637-5643.

24 H. Qian and R. Jin, Synthesis and electrospray mass spectrometry determination of thiolate-protected Au 55 (SR) 31 nanoclusters, Chem. Commun., 2011, 47, 11462-11464.

25 J. B. Tracy, M. C. Crowe, J. F. Parker, O. Hampe, C. A. Fields-Zinna, A. Dass and R. W. Murray, Electrospray ionization mass spectrometry of uniform and mixed monolayer nanoparticles: Au25 [S (CH2) 2Ph] 18 and Au25 [S (CH2) 2Ph] 18-x (SR) x, J. Am. Chem. Soc., 2007, 129, 16209-16215.

26 V. N. Popok, I. Barke, E. E. Campbell and K.-H. MeiwesBroer, Cluster-surface interaction: From soft landing to implantation, Surf. Sci. Rep., 2011, 66, 347-377.

27 P. Serra, M. Colina, J. M. Fernández-Pradas, L. Sevilla and J. L. Morenza, Preparation of functional DNA microarrays through laser-induced forward transfer, Appl. Phys. Lett., 2004, 85, 1639-1641.

28 S. Eibenberger, S. Gerlich, M. Arndt, M. Mayor and J. Tüxen, Matter-wave interference of particles selected from a molecular library with masses exceeding 10000 amu, Phys. Chem. Chem. Phys., 2013, 15, 14696-14700.

29 I. Tomalová, V. Frankevich and R. Zenobi, On initial ion velocities in MALDI: A novel FT-ICR MS approach, Int. J. Mass Spectrom., 2014, 372, 51-53.

30 U. Sezer, P. Schmid, L. Felix, M. Mayor and M. Arndt, Stability of high-mass molecular libraries: the role of the oligoporphyrin core, J. Mass. Spectrom., 2015, 50, 235-239.
31 Y. Chao, L. Šiller, S. Krishnamurthy, P. R. Coxon, U. Bangert, M. Gass, L. Kjeldgaard, S. N. Patole, L. H. Lie and N. O'Farrell, Evaporation and deposition of alkylcapped silicon nanocrystals in ultrahigh vacuum, Nat. Nanotechnol., 2007, 2, 486-489.

32 S. Deachapunya, P. J. Fagan, A. G. Major, E. Reiger, H. Ritsch, A. Stefanov, H. Ulbricht and M. Arndt, Slow beams of massive molecules, Eur. Phys. J. D, 2008, 46, 307313.

33 S. Gerlich, S. Eibenberger, M. Tomandl, S. Nimmrichter, K. Hornberger, P. J. Fagan, J. Tüxen, M. Mayor and M. Arndt, Quantum interference of large organic molecules, Nat. Commun., 2011, 2, 263.

34 L. Felix, U. Sezer, M. Arndt and M. Mayor, Synthesis of Highly Fluoroalkyl-Functionalized Oligoporphyrin Systems, Eur. J. Org. Chem., 2014, 6884-6895.

35 A. Desireddy, B. E. Conn, J. Guo, B. Yoon, R. N. Barnett, B. M. Monahan, K. Kirschbaum, W. P. Griffith, R. L. Whetten and U. Landman, Ultrastable silver nanoparticles, Nature, 2013, 501, 399-402.

36 M. Brust, M. Walker, D. Bethell, D. J. Schiffrin and R. Whyman, Synthesis of thiol-derivatised gold nanoparticles in a two-phase liquid-liquid system, J. Chem. Soc., Chem. Commun., 1994, 801-802.

37 S. R. K. Perala and S. Kumar, On the mechanism of metal nanoparticle synthesis in the Brust-Schiffrin method, Langmuir, 2013, 29, 9863-9873.

38 S. E. Skrabalak, B. J. Wiley, M. Kim, E. V. Formo and Y. Xia, On the polyol synthesis of silver nanostructures: glycolaldehyde as a reducing agent, Nano Lett., 2008, 8, 2077-2081.

39 Y. Sun, B. Mayers, T. Herricks and Y. Xia, Polyol synthesis of uniform silver nanowires: a plausible growth mechanism and the supporting evidence, Nano Lett., 2003, 3, 955-960.

40 R. Jin, C. Zeng, M. Zhou and Y. Chen, Atomically Precise Colloidal Metal Nanoclusters and Nanoparticles: Fundamentals and Opportunities, Chem. Rev., 2016, 116, 10346-10413.

41 M. E. Kurczy, Z.-J. Zhu, J. Ivanisevic, A. M. Schuyler, K. Lalwani, A. F. Santidrian, J. M. David, A. Giddabasappa, A. J. Roberts and H. J. Olivos, Comprehensive bioimaging with fluorinated nanoparticles using breathable liquids, Nat. Commun., 2015, 6, 5998.

42 T. Juffmann, A. Milic, M. Mullneritsch, P. Asenbaum, A. Tsukernik, J. Tuxen, M. Mayor, O. Cheshnovsky and M. Arndt, Real-time single-molecule imaging of quantum interference, Nat. Nanotechnol., 2012, 7, 297-300. 